\title{
Mesoniviridae: a proposed new family in the order Nidovirales formed by a single species of mosquito-borne viruses
}

\author{
Chris Lauber $\cdot$ John Ziebuhr $\cdot$ Sandra Junglen $\cdot$ \\ Christian Drosten · Florian Zirkel · Phan Thi Nga • \\ Kouichi Morita $\cdot$ Eric J. Snijder · Alexander E. Gorbalenya
}

Received: 20 January 2012/Accepted: 27 February 2012/Published online: 24 April 2012

(C) The Author(s) 2012. This article is published with open access at Springerlink.com

\begin{abstract}
Recently, two independent surveillance studies in Côte d'Ivoire and Vietnam, respectively, led to the discovery of two mosquito-borne viruses, Cavally virus and Nam Dinh virus, with genome and proteome properties typical for viruses of the order Nidovirales. Using a stateof-the-art approach, we show that the two insect nidoviruses are (i) sufficiently different from other nidoviruses to represent a new virus family, and (ii) related to each other closely enough to be placed in the same virus species. We propose to name this new family Mesoniviridae. Meso is derived from the Greek word "mesos" (in English "in the middle") and refers to the distinctive genome size of these
\end{abstract}

C. Lauber · E. J. Snijder · A. E. Gorbalenya $(\square)$

Molecular Virology Laboratory, Department of Medical

Microbiology, Leiden University Medical Center,

Albinusdreef 2, E4-P, P.O. Box 9600,

2300 RC Leiden, The Netherlands

e-mail: a.e.gorbalenya@lumc.nl

J. Ziebuhr

Institute of Medical Virology,

Justus Liebig University Giessen,

Giessen, Germany

S. Junglen $(\bowtie) \cdot$ C. Drosten $\cdot$ F. Zirkel

Institute of Virology, University of Bonn Medical Center,

Bonn, Germany

e-mail: junglen@virology-bonn.de

P. T. Nga

Department of Virology, National Institute of Hygiene and Epidemiology, Hanoi 100-000, Vietnam

K. Morita $(\bowtie)$

Department of Virology, Institute of Tropical Medicine,

Global COE Program, Nagasaki University,

Nagasaki 852-8523, Japan

e-mail: moritak@nagasaki-u.ac.jp insect nidoviruses, which is intermediate between that of the families Arteriviridae and Coronaviridae, while $n i$ is an abbreviation for "nido". A taxonomic proposal to establish the new family Mesoniviridae, genus Alphamesonivirus, and species Alphamesonivirus 1 has been approved for consideration by the Executive Committee of the ICTV.

The order Nidovirales [1] includes positive-sense singlestranded RNA (ssRNA+) viruses of three families: Arteriviridae [2] (12.7-15.7-kb genomes; "small-sized nidoviruses"), Coronaviridae [3] and Roniviridae [4] (26.3-31.7 kb; the last two families are jointly referred to as "large-sized nidoviruses") [5]. All other known ssRNA+ viruses have genome sizes below $20 \mathrm{~kb}$. Recently, two closely related viruses, Cavally virus (CAVV) and Nam Dinh virus (NDiV), were discovered by two independent groups of researchers in Côte d'Ivoire in 2004 and in Vietnam in 2002, respectively [6, 7]. CAVV was isolated from various mosquito species belonging to the genera Culex, Aedes, Anopheles and Uranotaenia [7]. It was most frequently found in Culex species, especially Culex nebulosus. Except for Culex quinquefasciatus, which circulates worldwide, the other mosquito species are endemic to Africa. NDiV was isolated from Culex vishnui, which is endemic to Asia, and Culex tritaeniorhynchus, which circulates in Asia and Africa [6], and there are indications that it may infect more mosquito species (Nga, unpublished data). Analysis of abundance patterns of $39 \mathrm{CAVV}$ isolates in different habitat types along an anthropogenic disturbance gradient has indicated an increase in virus prevalence from natural to modified habitat types [8]. A significantly higher prevalence was found especially in human settlements. Analysis of habitatspecific virus diversity and ancestral state reconstruction demonstrated an origin of CAVV in a pristine rainforest with 
subsequent spread into agriculture and human settlements [7]. Notably, it was shown for the first time that virus diversity decreased and prevalence increased during the process of emergence from a pristine rainforest habitat into surrounding areas of less host biodiversity due to anthropogenic modification [7]. Both viruses were propagated in Aedes albopictus cells and characterized using different techniques. A number of common properties place CAVV and NDiV in the order Nidovirales. These properties include (i) the genome organization with multiple open reading frames (ORFs), (ii) the predicted proteomes (Fig. 1), (iii) the production of enveloped, spherical virions, and (iv) the synthesis of genome-length and subgenome-length viral RNAs in infected cells [6, 7]. Particularly, the two viruses were found to encode key molecular markers characteristic of all nidoviruses: a 3C-like main protease (3CLpro, also known as Mpro) flanked by two transmembrane (tM) domains encoded in replicase ORF1a, as well as an RNAdependent RNA polymerase (RdRp) and a combination of a $\mathrm{Zn}$-binding module ( $\mathrm{Zm}$ ) fused with a superfamily 1 helicase (HEL1) encoded in ORF1b. As in other nidovirus genomes, ORFs $1 \mathrm{a}$ and $1 \mathrm{~b}$ were found to overlap by a few nucleotides in both CAVV and NDiV. The ORF1a/1b overlap region includes a putative -1 ribosomal frameshift site (RFS) that is expected to direct the translation of ORF1b by a fraction of the ribosomes that start translation at the ORF1a initiation codon. Thus, a frameshift just upstream of the ORF1a termination codon mediates the production of a C-terminally extended polyprotein jointly encoded by ORF1a and ORF1b. Combined, these markers form the characteristic nidovirus constellation: tM-3CLpro-tM_RFS_RdRp_ZmHEL1 (Fig. 1) [1, 5]. Likewise, virion proteins are encoded in ORFs that are located downstream of ORF1b and expressed from a set of subgenomic mRNAs. No similarities were found between the (putative) structural proteins of CAVV and NDiV and those of other nidoviruses [6, 7]. The most distinctive molecular characteristic of CAVV and $\mathrm{NDiV}$, however, is the $\sim 20$-kb genome size, that is intermediate between the size ranges of small-sized and largesized nidovirus genomes. Consequently, each of the two viruses has been proposed to prototype a new nidovirus family $[6,7]$.
In this study, we compared the genomes of CAVV (GenBank accession number HM746600) and NDiV (GenBank accession number DQ458789) to assess their relationship and use this insight for taxonomic classification of these viruses. To date, only very limited biological information is available for CAVV and NDiV (see above), and in general, biological properties may be affected profoundly by a few changes in the genome. In view of these considerations and in line with the accepted taxonomic approach to viruses of the family Coronaviridae [3], comparative sequence analysis was considered the most reliable basis for classification. The overall similarity between the CAVV and NDiV genomes was found to be strikingly high: nearly identical sizes (20,187 and 20,192 nt, respectively), conservation of ORFs with sequence identities ranging from 87.8 to $96.1 \%$ at the amino acid level and from 88.3 to $93.7 \%$ at the nucleotide level (Table 1). Given this high similarity, prior assignments of domains and genetic signals were cross-checked to produce a unified description.

There was complete agreement between the two studies $[6,7]$ on the mapping of all nidovirus-wide conserved domains in CAVV and NDiV, as well as on the identification of GGAUUUU as a plausible slippery sequence in RFS (see above). Additionally, our analysis showed that the NDiV-based assignment [6] of $3^{\prime}$-to- $5^{\prime}$ exoribonuclease (ExoN) and $2^{\prime}$-O-methyltransferase (OMT), two replicative domains characteristic for large-sized nidoviruses [5], and N7-methyltransferase (NMT) [9] in ORF1b extends to CAVV. Likewise, CAVV may lack a uridylate-specific endonuclease (NendoU), as has previously been observed for NDiV [6]. The synthesis of subgenomic RNAs from which ORFs $2 \mathrm{a}$ to 4 are predicted to be expressed appears to be controlled by transcription-regulating sequences (TRSs) [10-12] identified upstream of ORF2a/2b, ORF3a and ORF4 (collectively designated as body TRSs). Other putative TRSs were identified downstream of the leader region located at the $5^{\prime}$-end of the viral genome [6, 7]. Unique among nidoviruses, NDiV and CAVV may use different leader TRSs during the synthesis of different subgenomic RNAs, although further analysis is required to clarify the basis for some discrepancies between the TRS assignment in NDiV and CAVV. Also, it remains to be

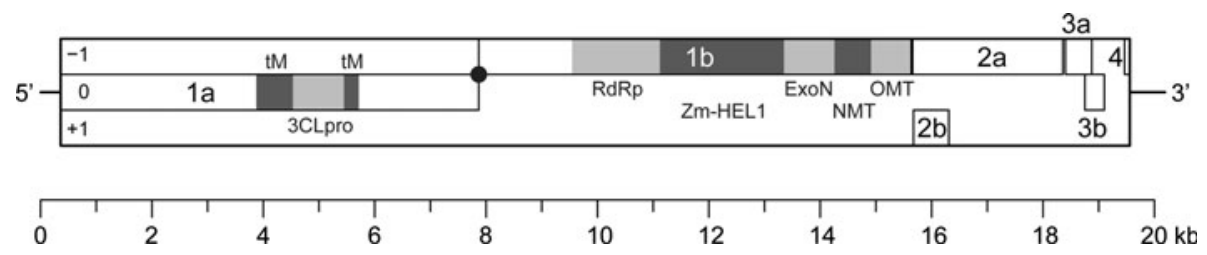

Fig. 1 Genome organization of mesoniviruses. The coding and 5'and $3^{\prime}$-untranslated regions of the genome are represented, respectively, by the outer rectangle and horizontal lines. ORFs are shown as open rectangles and are arranged in three reading frames $(-1,0,+1)$ relative to that of ORF1a. ORF1a- and ORF1b-encoded protein domains identified by bioinformatics analysis (see ref. [6]) are highlighted in grey. The predicted location of -1 ribosomal frameshift signals are indicated by a black dot. The genome organization is shown for NDiV but is virtually identical to that of CAVV except for the reading frame of some ORFs (see Table 1) 
Table 1 Comparison of ORFs in the genome of NDiV and CAVV

\begin{tabular}{|c|c|c|c|c|c|c|c|}
\hline & \multicolumn{2}{|c|}{ Length [nt] } & \multicolumn{2}{|c|}{ Frame $^{\mathrm{a}}$} & \multicolumn{2}{|c|}{ Identity [\%] ${ }^{\mathrm{b}}$} & \multirow[t]{2}{*}{ Predicted protein } \\
\hline & NDiV & CAVV & NDiV & CAVV & nt & aa & \\
\hline ORF1a & 7509 & 7497 & 0 & 0 & 88.3 & 90.0 & Polyprotein 1a \\
\hline ORF1b & 7587 & 7587 & -1 & -1 & 92.6 & 96.1 & $1 \mathrm{~b}$ part of polyprotein $1 \mathrm{ab}$ \\
\hline ORF2a & 2697 & 2700 & -1 & -1 & 90.7 & 87.5 & Spike \\
\hline ORF2b & 636 & 642 & +1 & +1 & 88.8 & 90.2 & Nucleocapsid \\
\hline ORF3a & 474 & 474 & -1 & +1 & 91.1 & 93.0 & Membrane \\
\hline ORF3b & 348 & 348 & 0 & -1 & 93.7 & 90.5 & Membrane \\
\hline ORF4 & 135 & 147 & +1 & -1 & 89.9 & 87.8 & Unknown \\
\hline
\end{tabular}

ORF designations according to Table 2 in ref. [7]

${ }^{a}$ Reading frame relative to that of ORF1a

${ }^{b}$ Pairwise nucleotide (nt) and amino acid (aa) sequence identity between NDiV and CAVV

shown why the high sequence conservation of virion proteins of the two viruses (Table 1) was not manifested in the morphology observed upon EM analysis of virus particles $[6,7]$. In this respect, it may be relevant that Zirkel et al. [7] noticed two types of particles in CAVV-infected cells, one of which carried club-shaped surface projections compatible with viral glycoproteins. This latter type of particles was also observed in infected cell culture supernatant. Ultimately, the origin of the particles of both types, and their relationship to the particles isolated from the medium of NDiV-infected C6/36 cells by Nga et al. [6] should be revealed by future research efforts.

Furthermore, we evaluated the phylogenetic position of CAVV and NDiV in relation to other nidoviruses. We conducted a phylogenetic analysis as described in ref. [6]. The study indicates that CAVV and NDiV consistently, albeit very distantly, cluster with viruses of the family Roniviridae, the only other known nidoviruses infecting invertebrates (Fig. 2). Quantitatively, this Bayesian posterior probability phylogeny illustrates that CAVV and NDiV form a deeply rooted lineage in the nidovirus tree with an evolutionary divergence from other nidoviruses comparable to that separating viruses of the families Coronaviridae and Roniviridae (Fig. 2). Together, these characteristics of CAVV and NDiV (insect host, intermediate genome size, deeply rooted phylogenetic lineage) provide a compelling basis for the creation of a new nidovirus family. We propose to name this new family Mesoniviridae, where meso is derived from the Greek word "mesos" (in English "middle" or "in the middle") and refers to a key distinctive characteristic of these viruses, namely their intermediate-sized genomes. The second component of the acronym, ni, refers to nidoviruses, as has been done previously for roniviruses [13] and bafiniviruses [14].

Next, we sought to establish species demarcation criteria to decide whether CAVV and NDiV prototype separate species or belong to a single species. Commonly, this question cannot be answered (reliably) on the basis of only two full genome sequences and otherwise very limited biological data. To solve this dilemma, we exploited information available for other nidoviruses in our analysis. In order to evaluate the genetic similarity between CAVV and NDiV in the context of sequence divergence of lineages representing previously established nidovirus species, we applied a state-of-the-art framework for a geneticsbased classification [15]. This recently introduced classification approach has been shown to recover and refine the taxonomy of picornaviruses [16], and it was also used to revise the taxonomy of coronaviruses extensively (Lauber \& Gorbalenya, in preparation) [3]. In addition to CAVV and NDiV, a representative set of 152 large-sized nidoviruses was included in the analysis. Two sets of proteins were used: the first included proteins conserved in all nidoviruses (3CLpro, RdRp, HEL1) (dataset D1), while the second set additionally included ExoN and OMT, which are conserved in large-sized nidoviruses and CAVV/NDiV (dataset D2). For both datasets a concatenated, multiple amino acid alignment was produced, which formed the basis for compiling pairwise evolutionary distances (PEDs) between all pairs of viruses (Fig. 3ab; for details see ref. [15]). It was found that the PED separating CAVV and $\mathrm{NDiV}$ is within the range of intra-species virus divergence in the families Coronaviridae and Roniviridae for both datasets (Fig. 3cd). Specifically, CAVV and NDiV show a distance (0.016 and 0.029 for D1 and D2, respectively) that is below the genetic divergence of members of several established nidovirus species (maximum of 0.032 and 0.037 for D1 and D2, respectively). For both datasets, these viruses include gill-associated virus and yellow head virus (species Gill-associated virus, family Roniviridae) [4] and the coronaviruses feline coronavirus, transmissible gastroenteritis virus, and porcine respiratory coronavirus (species Alphacoronavirus 1), IBV (species Avian coronavirus), murine hepatitis virus (species Murine coronavirus), and 


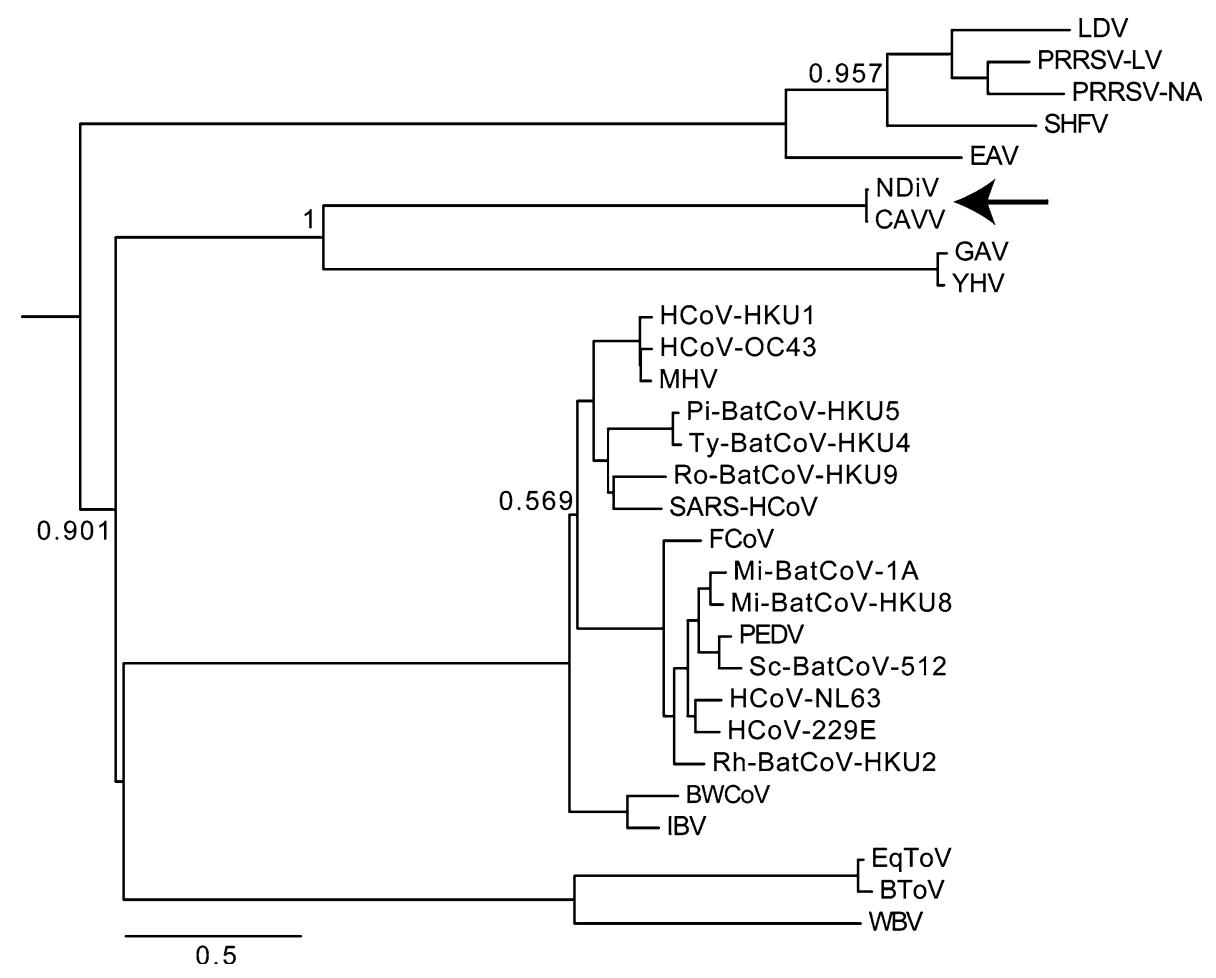

Fig. 2 Phylogenetic position of CAVV and NDiV. To infer phylogenetic relationships of Nam Dinh virus isolate 02VN178 (NDiV), Cavally virus isolate C79 (CAVV) (arrow) and other nidoviruses, a partially constrained tree was calculated using a concatenated alignment of the three nidovirus-wide conserved domains and a set of viruses representing currently recognized species. The alignment was produced with Muscle version 3.52 [17] in the Viralis platform [18], and the phylogenetic analysis was performed using BEAST version 1.4.7 [19]. For further details, see ref. [6]. Numbers indicate posterior probability support values (on a scale from 0 to 1); all internal nodes for which no support value is provided have been fixed in the analysis based on prior analyses of nidovirus subsets (data not shown). The scale bars represent the average number of substitutions per amino acid position. The tree was rooted on the arterivirus branch. Virus names and GenBank/Refseq accession numbers: lactate dehydrogenase-elevating virus (LDV; U15146), porcine respiratory and reproductive syndrome virus European type (PRRSV-LV; M96262), porcine respiratory and reproductive syndrome virus North American type (PRRSV-NA; AF176348), simian hemorrhagic fever virus (SHFV; NC_003092), equine arteritis virus (EAV; AY349167),

Rousettus bat coronavirus HKU9 (species Rousettus bat coronavirus HKU9) [3]. For the dataset comprising the three nidovirus-wide conserved proteins (Fig. 3ac), Miniopterus bat coronavirus 1 also showed a maximum genetic divergence exceeding that of the CAVV-NDiV pair. Together, these observations show that CAVV and NDiV belong to the same species, representing a single genus in the family. We propose to name this genus Alphamesonivirus and the species Alphamesonivirus 1, thereby following a naming convention recently applied to the subfamily Coronavirinae [3], which is expected to facilitate the accommodation of future expansions of the family. A taxonomic proposal for family, genus, and species
Nam Dinh virus (NDiV; DQ458789), Cavally virus (CAVV; HM746600), gill-associated virus (GAV; AF227196), yellow head virus (YHV; EU487200), human coronavirus HKU1 (HCoV-HKU1; AY884001), human coronavirus OC43 (HCoV-OC43; AY585228), mouse hepatitis virus (MHV; AY700211), Pipistrellus bat coronavirus HKU5 (Pi-BatCoV-HKU5; EF065509), Tylonycteris bat coronavirus HKU4 (Ty-BatCoV-HKU4; EF065505), Rousettus bat coronavirus HKU9 (Ro-BatCoV-HKU9; EF065513), SARS coronavirus (SARSHCoV; AY345988), feline coronavirus (FCoV; NC_007025), Miniopterus bat coronavirus 1A (Mi-BatCoV-1A; NC_010437), Miniopterus bat coronavirus HKU8 (Mi-BatCoV-HKU8; NC_010438), porcine epidemic diarrhoea virus (PEDV; NC_003436), Scotophilus bat coronavirus 512 (Sc-BatCoV-512; DQ648858), human coronavirus NL63 (HCoV-NL63; DQ445911), human coronavirus 229E (HCoV-229E; NC_002645), Rhinolophus bat coronavirus HKU2 (Rh-BatCoV-HKU2; NC_009988), beluga whale coronavirus SW1 (BWCoV; EU111742), avian infectious bronchitis virus (IBV; NC_001451), equine torovirus (EToV; X52374), bovine torovirus (BToV; NC_007447), white bream virus (WBV; NC_008516)

recognition has been available on-line at the ICTV website (http://talk.ictvonline.org/files/proposals/taxonomy_proposals_ invertebrate $1 / \mathrm{m} /$ default.aspx) since August 2011. It has been approved by the chairs of the ICTV Arteriviridae, Coronaviridae, and Roniviridae Study Groups and the Executive Committee of the ICTV, and will be considered again at the next EC-ICTV meeting, to be held in Leuven, Belgium, in July 2012.

The recognition of CAVV and NDiV as a single virus species can be contrasted with the detection of these viruses in many mosquito host species and their spread to different continents (Africa and Asia, respectively) [6, 7]. The underlying mechanisms of this broad dispersal are 


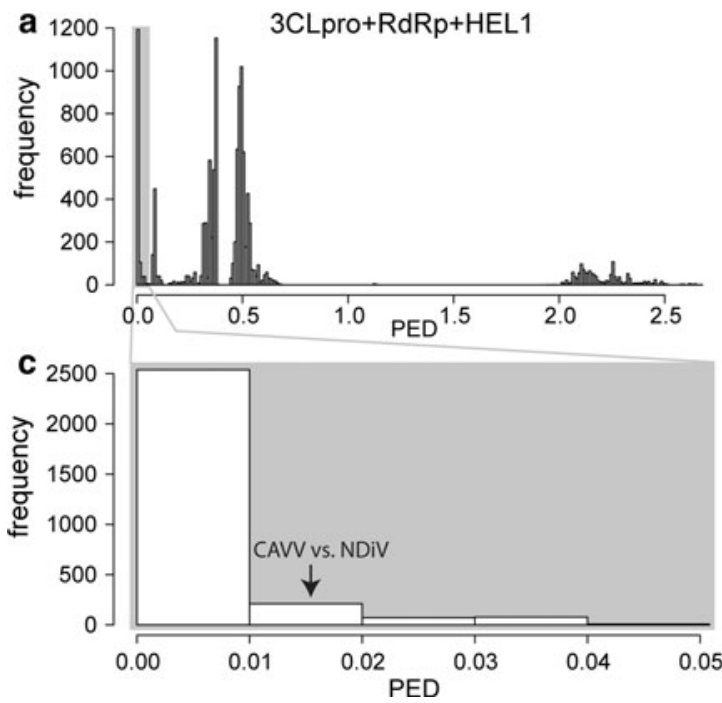

Fig. 3 Evolutionary distance between CAVV and NDiV in relation to intra-species genetic divergence in large-sized nidoviruses. Multiple amino acid alignments for 154 nidoviruses with large genomes (all major nidovirus lineages except arteriviruses) comprising three nidovirus-wide conserved protein domains $(\mathbf{a}, \mathbf{c})$ or five domains conserved in all large-sized nidoviruses $(\mathbf{b}, \mathbf{d})$ were used to compile pairwise evolutionary distances (PEDs) between all virus pairs. These

unknown but might include the crossing of the host species barrier rather than virus-host cospeciation. Further research, including the characterization of biological properties of CAVV and NDiV and the extension of surveillance studies to other regions of the world, is needed to understand the ecology, host tropism and medical and/or economic relevance of mesoniviruses.

Acknowledgments We thank Kay Faaberg, Jeff Cowley, and Raoul de Groot for reviewing the taxonomic proposal to establish the family Mesoniviridae that was used to draft this publication. Expert administration of the Viralis platform by Igor Sidorov, Alexander Kravchenko and Dmitry Samborskiy is acknowledged. This research has received funding from the European Union Seventh Framework Programme (FP7/2007-2013) under the programs SILVER (grant agreement no. 260644), EMPERIE (grant agreement number 223498), and EVA (grant agreement number 228292), and the Deutsche Forschungsgemeinschaft (grant agreement number DR772/ 3-1), LUMC MoBiLe program, Leiden University Fund, the BonFor programme of the University of Bonn (Grant agreement number O-156-0006), and the Program of Japan Initiative for Global Research Network on Infectious Diseases (J-GRID), MEXT, Japan.

Open Access This article is distributed under the terms of the Creative Commons Attribution License which permits any use, distribution, and reproduction in any medium, provided the original author(s) and the source are credited.

\section{References}

1. de Groot RJ, Cowley JA, Enjuanes L, Faaberg KS, Perlman S, Rottier PJM, Snijder EJ, Ziebuhr J, Gorbalenya AE (2012) Order Nidovirales. In: King AMQ, Adams MJ, Carstens EB, Lefkowitz

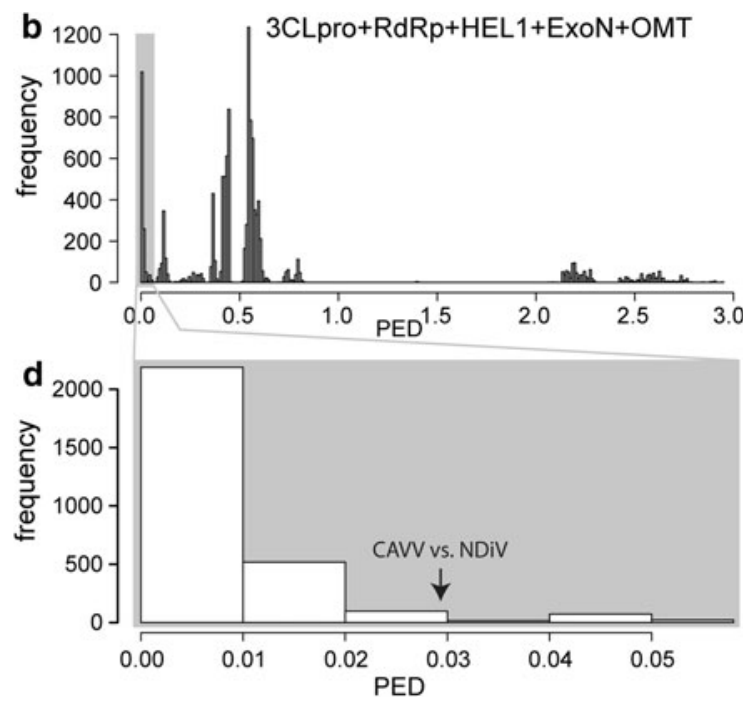

distances are shown as frequency distributions $(\mathbf{a}, \mathbf{b})$, and zoom-ins on small distances are provided $(\mathbf{c}, \mathbf{d})$. The PED between CAVV and $\mathrm{NDiV}$ (indicated by the arrow) is well within the intra-species distance range of other nidoviruses. Several currently recognized nidovirus species show a maximum genetic divergence larger than that of the CAVV-NDiV pair (see text)

EJ (eds) Virus taxonomy. Ninth report of the international committee on taxonomy of viruses. Elsevier Academic Press, Amsterdam, pp 785-795

2. Faaberg KS, Balasuriya UB, Brinton MA, Gorbalenya AE, Leung FC-C, Nauwynck H, Snijder EJ, Stadejek T, Yang H, Yoo D (2012) Family Arteriviridae. In: King AMQ, Adams MJ, Carstens EB, Lefkowitz EJ (eds) Virus taxonomy. Ninth report of the international committee on taxonomy of viruses. Elsevier Academic Press, Amsterdam, pp 796-805

3. de Groot RJ, Baker SC, Baric R, Enjuanes L, Gorbalenya AE, Holmes KV, Perlman S, Poon LL, Rottier PJM, Talbot PJ, Woo PCY, Ziebuhr J (2012) Family Coronaviridae. In: King AMQ, Adams MJ, Carstens EB, Lefkowitz EJ (eds) Virus taxonomy. Ninth report of the international committee on taxonomy of viruses. Elsevier Academic Press, Amsterdam, pp 806-828

4. Cowley JA, Walker PJ, Flegel TW, Lightner DV, Bonami JR, Snijder EJ, de Groot RJ (2012) Family Roniviridae. In: King AMQ, Adams MJ, Carstens EB, Lefkowitz EJ (eds) Virus taxonomy. Ninth report of the international committee on taxonomy of viruses. Elsevier Academic Press, Amsterdam, pp 829-834

5. Gorbalenya AE, Enjuanes L, Ziebuhr J, Snijder EJ (2006) Nidovirales: evolving the largest RNA virus genome. Virus Res 117:17-37

6. Nga PT, Parquet MD, Lauber C, Parida M, Nabeshima T, Yu FX, Thuy NT, Inoue S, Ito T, Okamoto K, Ichinose A, Snijder EJ, Morita K, Gorbalenya AE (2011) Discovery of the first insect nidovirus, a missing evolutionary link in the emergence of the largest RNA virus genomes. PLoS Pathog 7:e1002215

7. Zirkel F, Kurth A, Quan PL, Briese T, Ellerbrok H, Pauli G, Leendertz FH, Lipkin WI, Ziebuhr J, Drosten C, Junglen S (2011) An insect nidovirus emerging from a primary tropical rainforest. MBio 2:e00077-11

8. Junglen S, Kurth A, Kuehl H, Quan PL, Ellerbrok H, Pauli G, Nitsche A, Nunn C, Rich SM, Lipkin WI, Briese T, Leendertz FH (2009) Examining landscape factors influencing relative distribution of mosquito genera and frequency of virus infection. EcoHealth 6:239-249 
9. Chen Y, Cai H, Pan J, Xiang N, Tien P, Ahola T, Guo DY (2009) Functional screen reveals SARS coronavirus nonstructural protein nsp14 as a novel cap N7 methyltransferase. Proc Natl Acad Sci USA 106:3484-3489

10. Enjuanes L, Almazan F, Sola I, Zuniga S (2006) Biochemical aspects of coronavirus replication and virus-host interaction. Ann Rev Microbiol 60:211-230

11. Pasternak AO, Spaan WJM, Snijder EJ (2006) Nidovirus transcription: how to make sense...? J Gen Virol 87:1403-1421

12. Sawicki SG, Sawicki DL, Siddell SG (2007) A contemporary view of coronavirus transcription. J Virol 81:20-29

13. Cowley JA, Walker PJ (2002) The complete genome sequence of gill-associated virus of Penaeus monodon prawns indicates a gene organization unique among nidoviruses. Arch Virol 147:1977-1987

14. Schutze H, Ulferts R, Schelle B, Bayer S, Granzow H, Hoffmann B, Mettenleiter TC, Ziebuhr J (2006) Characterization of White bream virus reveals a novel genetic cluster of nidoviruses. J Virol 80:11598-11609
15. Lauber C, Gorbalenya AE (2012) Partitioning the genetic diversity of a virus family: approach and evaluation through a case study of Picornaviruses. J Virol 86:3890-3904

16. Lauber C, Gorbalenya AE (2012) Toward genetics-based virus taxonomy: comparative analysis of a genetics-based classification and the taxonomy of picornaviruses. J Virol 86:3905-3915

17. Edgar RC (2004) MUSCLE: a multiple sequence alignment method with reduced time and space complexity. BMC Bioinformatics 5:113

18. Gorbalenya AE, Lieutaud P, Harris MR, Coutard B, Canard B, Kleywegt GJ, Kravchenko AA, Samborskiy DV, Sidorov IA, Leontovich AM, Jones TA (2010) Practical application of bioinformatics by the multidisciplinary VIZIER consortium. Antiviral Res 87:95-110

19. Drummond AJ, Rambaut A (2007) BEAST: Bayesian evolutionary analysis by sampling trees. BMC Evol Biol 7:214 